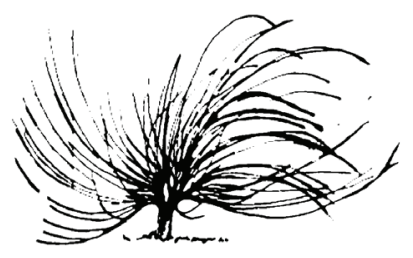

\title{
Percepción sistémica de la innovación educativa: Reflexiones desde el nuevo paradigma científico
}

\author{
Giovanni Sánchez Chacón ${ }^{1}$ \\ Universidad Nacional \\ Heredia, Costa Rica \\ giovanni.sanchez.ch@gmail.com
}

\begin{abstract}
Resumen
El presente ensayo presenta una reflexión en torno a los aportes del nuevo paradigma científico y el pensamiento sistémico que, transferidos al ámbito de la educación, podrían aportar herramientas de interés en el desarrollo de una comprensión profunda y holística de la complejidad subyacente al hecho educativo y, concretamente, del papel relevante que la percepción sistémica de la innovación podría asumir en este escenario.
\end{abstract}

Palabras clave: Pensamiento sistémico, nuevo paradigma científico, educación, innovación educativa, ecología.

\section{(c) (i) $\Theta$}

Recibido: 31 de julio de 2015-Aprobado: 25 de abril de 2016

1 Doctor en Psicología de la Educación, Cultura y Sistemas Semióticos. Universidad Autónoma de Barcelona, España. Posgrado en Psicología Evolutiva y de la Educación. Universidad Autónoma de Barcelona, España. Máster en Musicoterapia. Instituto Superior de Estudios Psicológicos, Barcelona, España. Máster en Psicopedagogía. Universidad Estatal a Distancia, UNED, Costa Rica. Licenciado en Ciencias de la Educación con énfasis en Didáctica de la Música. Universidad Nacional, Costa Rica. Bachiller en Música con énfasis en Educación Musical. Universidad Nacional, Costa Rica. 


\begin{abstract}
This essay presents a reflection on the contributions of the new scientific paradigm and systemic thinking, which transferred to the education field, could provide tools of interest in the development of a deep and holistic understanding of the underlying complexity of the educational processes and specifically the important role that systemic perception of innovation processes could assume in this context.
\end{abstract}

Keywords: systemic thinking, new scientific paradigm, educational innovation, education, ecology.

\title{
Introducción
}

Comprender la complejidad de la educación y, concretamente, de los procesos de innovación que en este contexto se generan representa una necesidad de cara a la consolidación de una educación holística, capaz de formar aprendices permanentes, tanto en ámbitos académicos, como en otros escenarios vinculados al desarrollo de competencias genéricas y otras trayectorias personales de aprendizaje que la vida misma demande.

Las aportaciones del nuevo paradigma científico y de la teoría de sistemas aportan herramientas conceptuales, procedimentales y de carácter condicional y estratégico que, transferidas a la educación, arrojan luces que permiten comprender la realidad y funcionamiento de la educación como un sistema complejo en el que entran en juego una serie de variables interconectadas e interdependientes. Comprender las diversas dinámicas que asumen dichas variables en determinados problemas propios de la educación y ser consciente de la complejidad presente en el hecho educativo permite el desarrollo de una nueva comprensión con respecto a este mismo, que redunde en el planteamiento de innovaciones educativas que atiendan, de manera más holística, los desafíos que la educación atraviesa.

En el presente ensayo se abordan, en primera instancia, conceptos claves en torno a al nuevo paradigma científico y cómo estos permiten comprender la realidad y los sistemas vivos desde una perspectiva global, sistémica e integradora. Dichos argumentos se sustentan a partir de los hallazgos obtenidos por diversos desarrollos científicos que, en los últimos 100 años, han venido transformando la visión mecanicista 
del mundo, de la realidad e incluso del fenómeno social y humano presente en el hecho educativo. En un segundo momento, dichos conceptos serán abordados en referencia a la complejidad sistémica implícita en la educación, argumentando la necesidad del desarrollo de una comprensión profunda de su naturaleza y de la conciencia con respecto al cómo distintas iniciativas de innovación deberían ser estratégicamente introducidas.

\section{Un nuevo paradigma científico}

Gran parte de las creencias que conforman la cultura general de una civilización usualmente están basadas en los paradigmas científicos predominantes en determinadas épocas de la historia. En este marco, Lipton (2009) señala que el antiguo paradigma saliente fundamentaba su concepción de mundo en la física newtoniana, en la cual solo a través del conocimiento de las leyes que dominan la materia se podría tener acceso a la comprensión profunda de la realidad, mientras que el nuevo paradigma emergente está amparado en la física cuántica, al comprender esta el mundo subatómico como un campo energético antimateria, de amplias posibilidades y controlado por leyes que distan mucho de las planteadas por Newton.

A nivel general, podemos afirmar que este nuevo paradigma científico considera la naturaleza como un sistema dinámico y reconoce al planeta como un ser vivo (hipótesis GAIA), capaz de auto organizarse. De acuerdo con Sahtouris (1994), las aportaciones de este paradigma no son del todo nuevas, ya que retoma algunas ideas existentes durante la Edad Media, el Renacimiento y durante la Ilustración, concretamente en la tradición alquímica. También sus aportaciones poseen una compatibilidad palpable con ciertos conceptos filosóficos y humanísticos que diversas tradiciones espirituales antiguas han sugerido milenios atrás.

De acuerdo con Capra y Steindl- Rast (1993), un paradigma podría definirse como una constelación de logros (conceptos, valores, técnicas, etc.) compartidos por una comunidad científica y empleados por esta para definir problemas y soluciones legítimas en torno a un ámbito de conocimiento.

Aunque las bases de este nuevo paradigma se instalaron en la primera mitad del siglo XX, en las últimas décadas un número importante de científicos y científicas han abrazado esta nueva visión de mundo y 
de la ciencia, renunciado al viejo paradigma, basado en una ecología mecanicista, superficial, reduccionista y antropocéntrica sobre la vida y la existencia. Al respecto, Rinaldi (2003) argumenta que este antiguo paradigma asume que el conocimiento del funcionamiento de las partes de un fenómeno es suficiente para comprender la complejidad del todo, y esta es la razón por la que el mismo desintegra, divide, reduce, fracciona, separa y fragmenta. Estos principios han sido considerados como parte de una ecología superficial; mientras que el nuevo paradigma sienta las bases de una revolución científica basada en una percepción existencial radicada en una ecología profunda, término atribuido a Naess (1973), el cual sostenía que los seres humanos, al igual que todos los sistemas vivos, coexisten y se automantienen a través de una red de relaciones de interacción e interdependencia.

Los aportes que emergen del nuevo paradigma científico, sin lugar a duda, impregnan de un profundo impacto a la educación y la pedagogía. Esta nueva concepción de la vida, que el nuevo paradigma comporta, asume una comprensión de carácter científico y holístico, tanto de la vida, como del ser humano y del universo. Así, pues, Rinaldi (2003) señala que, a diferencia del antiguo, este nuevo paradigma es organicista o naturalista, al considerar la naturaleza como un sistema orgánico y al reconocer al plantea como un ser vivo, tal y como lo asume la hipótesis GAIA. En general, este paradigma sustituye la visión antropocéntrica y competitiva de la vida, por una más cosmológica, cooperativa y democrática, rescatando la trascendencia de la experiencia humana, la espiritualidad y las emociones vinculadas al amor.

Como se ha visto, este nuevo paradigma no se limita al conocimiento del territorio, sino que pretende comprender, con mayor precisión, la topografía general del mapa, (naturaleza de la vida), ya que al percibir patrones subyacentes, reconoce que el todo es más que la suma de las partes y que cada parte es un todo en sí misma capaz de autoorganizarse. Siguiendo a Rinaldi (2003), se presenta la tabla 1 con una comparación de las características de los paradigmas saliente y entrante. 
Tabla 1. Características de los paradigmas saliente y entrante

\begin{tabular}{|l|l|l|}
\hline \multicolumn{1}{|c|}{ ANTIGUO PARADIGMA } & \multicolumn{1}{c|}{ NUEVO PARADIGMA } \\
\hline 1 & Mecanicista & Organicista, naturalista \\
\hline 2 & Desintegra, reduce, fracciona y separa & $\begin{array}{l}\text { Integra, multiplica, suma, unica, } \\
\text { crea, relaciona, conecta y totaliza }\end{array}$ \\
\hline 3 & $\begin{array}{l}\text { Especialista y desintegra el } \\
\text { conocimiento }\end{array}$ & $\begin{array}{l}\text { Sintético e integra el conocimiento en } \\
\text { redes transdisciplinarias }\end{array}$ \\
\hline 4 & Atiende a la parte & Atiende al todo \\
\hline 5 & Estudia objetos & Estudia relaciones \\
\hline 6 & $\begin{array}{l}\text { Entiende la relación causa - efecto } \\
\text { como un proceso línea }\end{array}$ & $\begin{array}{l}\text { Entiende las relaciones como } \\
\text { interconexiones no lineales }\end{array}$ \\
\hline 7 & Ritmos mecánicos & Ritmos naturales \\
\hline 8 & Rígido y estático & Flexible y dinámico \\
\hline 9 & Ordena y organiza & $\begin{array}{l}\text { Valora el caos como parte del proceso } \\
\text { creativo y de los cambios evolutivos } \\
\text { (auto organiza) }\end{array}$ \\
\hline 10 & Determinista & $\begin{array}{l}\text { Acepta la incertidumbre, lo } \\
\text { imprevisible y el misterio }\end{array}$ \\
\hline 11 & Crea certidumbres ficticias & Crea incertidumbre \\
\hline 12 & Cree en la acción & Contempla la acción - reacción \\
\hline 13 & $\begin{array}{l}\text { Antropocéntrico: relacionado con la } \\
\text { ecología superficial }\end{array}$ & $\begin{array}{l}\text { Visión cósmica: relacionado con la } \\
\text { ecología profunda }\end{array}$ \\
\hline 14 & Niega toda contradicción o la ignora & $\begin{array}{l}\text { Acepta la contradicción en la forma } \\
\text { de opuestos complementarios } \\
\text { creativos }\end{array}$ \\
\hline 15 & Individualiza & Colectivo, comunitario \\
\hline 16 & Patriarcal: dominante y competitivo & $\begin{array}{l}\text { Matriarcal: sin ser feminista, } \\
\text { democrático y cooperativo }\end{array}$ \\
\hline 17 & Acentúa el poder, el hacer & Acentúa el ser, el amar \\
\hline 18 & Intelectual, racional, lógico & $\begin{array}{l}\text { Reconoce la inteligencia emocional, } \\
\text { valora la intuición y es analógico }\end{array}$ \\
\hline 19 & Crítico & Autocrítico \\
\hline 20 & Materialista & Espiritual \\
\hline 21 & Preocupado por la forma, la \\
apariencia y la exteriorización & $\begin{array}{l}\text { Preocupado por el fondo, la esencia y } \\
\text { la interiorización }\end{array}$ \\
\hline 22 & Cuantitativo, mide & Cualitativo, mapea \\
\hline 23 & Modelo consumista, ser es tener & $\begin{array}{l}\text { Modelo autosustentable, } \\
\text { anticonsumista, ser es mucho más que } \\
\text { tener }\end{array}$ \\
\hline
\end{tabular}

Nota: (Rinaldi, 2003). 


\section{Desarrollos científicos en torno al nuevo paradigma}

Actualmente, existe un amplio número de disciplinas científicas que han contribuido, con sus aportes, a la conformación de esta nueva comprensión de la vida, cuyas implicaciones en el desarrollo de otros ámbitos del conocimiento han sido notorias en las últimas décadas. Al respecto, Capra (1998) argumenta que este paradigma centra su atención en el desarrollo de una nueva comprensión científica de la vida en todos los niveles de los sistemas vivientes, basándose en una nueva percepción de la realidad con profundas implicaciones no solo para la ciencia y la filosofía, sino también para los negocios, la política, la salud, la vida cotidiana e inclusive la educación. Tomando como base las aportaciones de Rinaldi (2003), a continuación se presenta la tabla 2, en la cual se exponen brevemente algunos desarrollos científicos pioneros y contemporáneos que han contribuido a la construcción científica de esta nueva visión de la vida.

Tabla 2. Primeros desarrollos científicos

\begin{tabular}{|l|l|}
\hline Biología & $\begin{array}{l}\text { Teoría de los campos morfogenéticos resonantes o hipótesis } \\
\text { de la causación formativa }\end{array}$ \\
\hline Evolución & Teoría del equilibrio puntuado \\
\hline Físico - química & Física cuántica, teoría holográfica, teoría del caos \\
\hline Geobiología & Teoría de GAIA \\
\hline Matemática & Geometría fractal, teoría de las catástrofes \\
\hline Teorías de unificación & Sinergética y teoría general de sistemas \\
\hline Psicología & Estudios sobre percepción aportados por la psicología gestalt \\
\hline $\begin{array}{l}\text { Psicología } \\
\text { transpersonal }\end{array}$ & $\begin{array}{l}\text { Aspectos espirituales involucrados en la transcendencia de } \\
\text { la experiencia humana }\end{array}$ \\
\hline Psiquiatría & $\begin{array}{l}\text { Recuerdos de existencias precedentes a partir de experiencias } \\
\text { de hipnosis clínica, estudio de casos y entrevistas a niños/as } \\
\text { entre 3 a 5 años }\end{array}$ \\
\hline $\begin{array}{l}\text { Tanatología y } \\
\text { cuidados paliativos }\end{array}$ & $\begin{array}{l}\text { Aportes realizados por estudios sobre experiencias cercanas } \\
\text { a la muerte y evidencia de vida después de la vida }\end{array}$ \\
\hline
\end{tabular}

Nota: (Rinaldi, 2003).

Después de esta reseña general aportada por la figura 2, a continuación se expondrán, de manera más amplia, los principios de algunos de estos desarrollos científicos que contribuyeron, en su momento y en 
la actualidad, en el establecimiento de las bases conceptuales de este nuevo paradigma.

\section{Física moderna}

Con los avances de la física moderna, a inicios del siglo XX, en la física de la época se empezó a experimentar cómo los principales fundamentos en los cuales estaba posicionada empezaban a perder sustento ante la nueva evidencia de la realidad subatómica. Al respecto, Bohr (1943) manifestaba:

La gran tensión que hemos soportado durante los últimos años, ha demostrado la insuficiencia de nuestras simples concepciones mecanicistas, y como consecuencia de ello, ha hecho que se tambaleen los cimientos sobre los que estaba basada la interpretación usual de nuestras observaciones. (p. 2)

Fueron los hallazgos en torno a la teoría de la relatividad y el de la física atómica los que vinieron a desmembrar las ideas cimentadas en la percepción newtoniana del mundo, en donde prevalecían la concepciones sobre las partículas elementales sólidas, la naturaleza causal de los fenómenos físicos, el espacio tiempo absoluto y, en general, la idea de una descripción meramente objetiva de los fenómenos de la naturaleza. Dentro de este escenario, fue Albert Einstein, a inicios del siglo pasado, quien sentó las bases de la física moderna, concretamente con los principios expuestos en su teoría de la relatividad y sus hallazgos en torno a la radiación electromagnética, lo cual se convertiría, años después, en las bases de la mecánica cuántica, la cual centró su atención en la investigación de la física del átomo y, concretamente, en la dinámica de las partículas subatómicas. En el marco de estos estudios, la física de la época dio con importantes hallazgos que posteriormente se convertirían en una de las evidencias científicas que darían origen a este nuevo paradigma. A nivel muy general, algunos de estas observaciones se exponen a continuación:

a. $\quad$ El $99.9 \%$ del volumen de los átomos que componen la materia representa un espacio vacío. 
b. Las partículas subatómicas se comportan como un sistema autoorganizado, pudiendo desdoblarse y hallarse en diferentes sitios de forman simultánea (desdoblamiento de las partículas).

c. Dichas partículas forman conjuntos de partículas entrelazadas y constituidas como un sistema, que con una función de onda, establecen relaciones de interconexión e interdependencia (entrelazamiento cuántico).

d. Dichas partículas, tras atravesar una bifurcación, pueden experimentar la coexistencia en distintos estados y realidades (paradoja de Schrödinger).

e. A nivel cuántico, no puede existir un fenómeno que pueda manifestarse físicamente hasta que este mismo sea observado. Desde esta perspectiva, el universo puede existir por el hecho de ser conscientes de su existencia (fenómeno observador).

Los anteriores datos causaron un gran asombro en la física de la época, la cual hasta la fecha, estaba cimentada en un paradigma mecanicista que consideraba el estudio de la materia como la única fuente de interpretación de la realidad. Al considerar que dichas partículas podrían asumir la posición de los "ladrillos" microscópicos de los que está compuesta la materia, es importante destacar su dinámica sorprendente de automantenimiento y autoorganización, lo cual debería tener implicaciones también de cara al conocimiento profundo de nuestra verdadera naturaleza humana.

\section{Biología organicista}

Otro ámbito de conocimiento que aportó datos y experiencias de investigación en la conformación del nuevo paradigma corresponde al campo de la biología organicista, en el cual se destacó como característica clave la naturaleza jerárquica de la organización de los organismos vivos. Dichas conclusiones son enfáticas en señalar que toda manifestación de vida se constituye a partir estructuras multinivel de sistemas dentro de sistemas cada vez más complejos. De acuerdo con Capra (1998), cada sistema forma un todo con respecto a sus partes y es, al mismo tiempo, parte de un todo más global. Siguiendo al mismo autor, vemos que las células se combinan para formar tejidos, estos para formar órganos y estos, a su vez, para formar organismos, los cuales 
coexisten dentro de ecosistemas y sistemas sociales. De lo anterior se infiere que en donde quiera que haya vida, encontraremos sistemas vivos coexistiendo dentro de otros más complejos, produciendo redes de sistemas interconectados e interdependientes.

Toda esta visión se nutrió en su momento con los aportes de los científicos chilenos Maturana y Varela, quienes en la década de los 70 denominaron con el nombre de "autopoiesis" a la química de producción, regeneración y automantenimiento de los sistemas vivos. Desde esta óptica, "auto" posee una connotación de referencia a "sí mismo" que pone en evidencia la autonomía de los sistemas autoorganizadores y, por otro lado, "poiesis", pertenece a la raíz griega "poesía" que significa "creación". De esta manera, el término autopoiesis está íntimamente vinculado con la creación de sí mismo como principal característica de los sistemas vivos.

Como veremos más adelante, dichos conceptos han sido empleados posteriormente por la teoría de sistemas y también transferidos al área de la sociología, con el propósito de trazar una relación entre estos y los procesos sociológicos implicados en el auto mantenimiento y organización de los seres humanos en la sociedad.

\section{Ecología profunda}

La palabra ecología proviene del griego "oikos", cuyo significado es "casa". Esta concibe el planeta tierra como un hogar, centrando su atención en las relaciones que vinculan a sus habitantes. Por su parte, Capra (1998) señala que la ciencia de la ecología emergió de la escuela organicista de biología durante el siglo XIX, justo en el momento en que se comenzó a estudiar las comunidades de organismos. Todo este movimiento fue impulsado, en primera instancia, por el biólogo alemán Ernst Haeckel, quien definía la ecología como la ciencia de las relaciones entre los organismos y el mundo exterior que le rodea.

Más adelante, Naes (1973) plantea el termino ecología profunda, el cual considera los organismos como nodos en la red biosférica o campo de relaciones intrínsecas. Es decir, sostiene que todos los organismos vivos están articulados a partir de relaciones de interconexión e interdependencia.

Es importante destacar que mientras la ecología confirmaba estos principios en sus estudios sobre comunidades de animales y plantas, la 
biología organicista estudiaba la totalidad irreductible en los organismos; la física cuántica, la dinámica de las partículas subatómicas, y la psicología gestalt, los fenómenos perceptivos.

\section{Pensamiento sistémico}

Las diversas percepciones de carácter reduccionista de los fenómenos de la vida han sido también un tema de discusión cuando se trata de reflexionar en torno a la educación. En las últimas décadas, diversas corrientes vinculadas a la pedagogía crítica y la sociología de la educación han manifestado su preocupación por el estudio de los fenómenos educativos desde una visión mecanicista y fragmentada.

Como se ha comentado, durante el pasado siglo se generó un movimiento científico que emigró del antiguo paradigma mecanicista, centrado en el estudio de las partes de un fenómeno, al ecológico, el cual se orienta al estudio de los fenómenos como un todo, asumiéndolos desde una perspectiva global, holística e integradora. La fuerte disputa entre el estudio de las partes y el todo, y el énfasis en el estudio de las partes como principal metodología para comprender los fenómenos de la vida se ha tornado una discusión importante también en el campo educativo, en donde diversas líneas de pensamiento asumen miradas cada vez más holísticas y, en contraste, otras hacia la comprensión del funcionamiento de las partes.

Refiriéndose a esta temática y desde la óptica del nuevo paradigma científico, Capra (1998) señala:

La tensión básica se da entre las partes y el todo. El énfasis sobre las partes se ha denominado mecanicista, reduccionista o atomista, mientras que el énfasis sobre el todo recibe los nombres de holístico, organicista o ecológico. En la ciencia del siglo XX la perspectiva holística ha sido conocida como «sistémica» y el modo de pensar que comporta como pensamiento sistémico. (p. 37)

Como se ha planteado, el pensamiento sistémico representa uno de los principales fundamentos del nuevo paradigma científico. Sus bases conceptuales surgen en el marco de diversas disciplinas, que en las cercanías de la segunda década del siglo XX y lideradas en principio por profesionales de la biología, física y psicología Gestalt, sentaron las 
bases de una visión de los organismos vivos como totalidades integradas interconectadas e interdependientes.

\section{Características del pensamiento sistémico}

Como se ha comentado, dentro de los principales objetivos del pensamiento sistémico se encuentra el desarrollo de una percepción de la realidad en términos de totalidades integradas. Su visión es distinta de los principios del método científico tradicional, el cual solo percibe partes de un fenómeno, usualmente de forma inconexa. Sus bases conceptuales se construyen a partir de los cuestionamientos desarrollados por Ludwing Von Bertalanffy, quien, desde el campo de la biología, cuestionó la aplicación del método científico en los problemas propios de la biología, ya que estos hasta la fecha eran interpretados desde una óptica mecanicista y causal, por lo cual carecían de la suficiente profundidad para la comprensión de los problemas fundamentales que se presentan en los sistemas vivos.

Estos principios podemos aplicarlos también a los distintos niveles de sistemas, entendidos como la dinámica de una ciudad, la economía o a fenómenos de carácter social y humano propios de la educación. De acuerdo con Capra (1998), el uso del pensamiento sistémico posee unos criterios claves, que permitirían comprender de manera más holística la naturaleza de los fenómenos sociales y humanos inmersos en el hecho educativo. Dichos criterios se exponen a continuación:

a. En definitivo, el principio más visible es el cambio de percepción de los fenómenos de las partes al todo. Desde esta perspectiva, tanto los sistemas vivos como de la mayoría de los fenómenos sociales y estrictamente humanos, como el caso de la educación, representan totalidades integradas cuyas propiedades no pueden ser reducidas a sus partes más pequeñas. Se asume, así, una posición holística, global e integradora en cuanto al estudio de los fenómenos de la vida.

b. Dichas propiedades emergen de las relaciones organizadoras que actúan sobre las partes, generando patrones de relaciones ordenadas que caracterizan aquella clase específica de organismos o sistemas. 
c. Las propiedades sistémicas quedan inútiles cuando el sistema se divide en elementos aislados.

d. El pensamiento sistémico asume la habilidad de concentrar la atención de manera simultánea en distintos niveles sistémicos, ya que en el caso de los sistemas vivos nos encontramos con sistemas dentro de sistemas.

e. Cada nivel sistémico comporta diversos niveles de complejidad. Es decir, en cada nivel, los fenómenos observados poseen propiedades que no se dan a niveles inferiores, los cuales se denominan niveles emergentes.

f. El pensamiento sistémico señala que los sistemas vivos no pueden ser comprendidos desde el análisis de sus partes, solo pueden entenderse desde el contexto global. Por tanto, el pensamiento sistémico es un pensamiento contextual que promueve una percepción profunda de lo ecológico.

g. Dentro de esta perspectiva y con base en los aportes de la física cuántica, las partes no existen en absoluto. Lo que concebimos como parte, realmente funciona como un patrón dentro de una inseparable red de relaciones interconectadas e interdependientes. De ahí que el cambio perceptivo de las partes al todo podría ser interpretado, también, como el cambio de objetos a relaciones.

h. En la visión mecanicista, el mundo es una colección de objetos; pero desde la perspectiva sistémica podemos observar que los objetos en sí mismos representan redes de relaciones inmersas en redes mayores, de ahí que pensar en relaciones es fundamental dentro de esta visión.

i. La percepción de la realidad como una red de relaciones interconectadas ha influenciado, no solo nuestra visión de la naturaleza, sino también el modo en el que se concibe el conocimiento científico, ya que este mismo formaría parte también de una red interconectada de conceptos y modelos en los que no existen cimientos, sino patrones y relaciones.

Como vemos, el pensamiento sistémico implica percibir la vida y sus fenómenos en forma de redes de sistemas interconectados a otros cada vez más complejos y ser capaz de observar los diversos patrones e interrelaciones que desde esta dinámica se generan. 


\section{Psicología gestalt}

La palabra alemana para denominar una forma orgánica es gestalt. La psicología de la gestalt fue fundada poco antes de la primera guerra mundial, por Wertheimer, Kohler y Koffka. Esta mantuvo una reacción de oposición en contra de la psicología tradicional que imperaba a principios del siglo XX. Desde esta vertiente, se optó por el estudio de las configuraciones y patrones naturales suscitados en la experiencia humana, en vez de reducirse solo al campo del estudio de la mente.

La escuela gestalt hizo contribuciones substanciales a la psicología, especialmente en el estudio y aprendizaje de la naturaleza de las asociaciones de carácter perceptivo, ya que en sus orígenes su campo de acción se orientó al estudio de la percepción, lo cual posteriormente se amplía a otros ámbitos vinculados a la conducta social, la educación y el desarrollo del pensamiento.

De acuerdo con Castanedo (1997), la psicología de la gestalt es conocida a su vez como teoría de campo, ya que esta considera que la conducta humana tiene lugar en el contexto de un medio ambiente conformado por un organizado sistema de tensiones y esfuerzos análogos que se dan dentro de un campo gravitacional o en un campo electromagnético.

Dese esta perspectiva, el filósofo Christian von Ehrenfelds señalaba que la gestalt asumía una posición en donde el todo es más que la suma de las partes, lo cual, como vimos, representó también un concepto clave en el pensamiento sistémico.

Capra (1998) señala que los psicólogos Gestalt pioneros afirmaban que los organismos vivos perciben la realidad no en términos de elementos aislados, sino de patrones perceptuales integrados o conjuntos organizados dotados de significado, los cuales exhiben cualidades ausentes en sus partes. Así pues, tanto la biología organicista como la gestalt concebían su escuela como una tercera línea de pensamiento que rebasaba los límites del mecanicismo.

\section{Psiquiatría y psicología transpersonal}

Durante los últimos 50 años y desde áreas más próximas a las ciencias sociales y de la salud, se han realizado aportes con un grado importante de compatibilidad con los principios de este nuevo paradigma. En el campo de la psiquiatría, se destaca el trabajo de los doctores 
norteamericanos Ian Stevenson, Raymond Moody, Brian Weiss, y Michael Newton, quienes, empleando técnicas avanzadas de hipnosis clínica, estudios de casos y entrevistas en profundidad a niños y niñas entre 3 y 5 años, realizaron importantes contribuciones con respecto a la naturaleza de la vida humana y de la vida después de la vida. Caso similar ocurrió con los hallazgos de la doctora suiza Elizabet Kübbler Ross, quien dedicó la mayor parte de su carrera al estudio científico de las experiencias cercanas a la muerte, realizando importantes aportaciones que, al día de hoy, están presentes dentro de diversos programas de estudio universitarios vinculados a las especialidades de psiquiatría, tanatología y cuidados paliativos.

A nivel general, los datos señalados por estos estudios revelan que al parecer, también en los seres humanos, la dinámica de la vida y la existencia posee una estructura sistémica y funciona a partir de relaciones de interdependencia e interconexión con otros "yoes" que coexisten en otras realidades del espacio tiempo, fenómeno definido por Roberts (1998) y Merlo (2007) como la naturaleza multidimencional del ser humano, lo cual representaría, dentro de esta visión, una aproximación a la concepción moderna y científica de la reencarnación. Las anteriores afirmaciones reflejan coherencia con ciertas aportaciones señalas por algunos físicos modernos en materia de desdoblamiento del tiempo, entrelazamiento cuántico y el estudio acerca de las dimensiones que conforman el cosmos.

Como se podrá ver, dichas conclusiones guardan también una cierta relación con algunas enseñanzas espirituales antiguas vinculadas con el hinduismo, budismo e incluso con la tradición mística del cristianismo primitivo, las cuales creían en la inmortalidad del alma y en su encarnación sucesiva en distintos cuerpos físicos, con el propósito de alcanzar, en este recorrido, conciencia de su naturaleza trascendente y una mayor evolución espiritual. Cabe destacar que todas estas aportaciones, que empiezan a establecer un camino de reconciliación entre ciencia y espiritualidad, también guardan una relación estrecha con los aportes de la psicología transpersonal, cuyas bases fueron establecidas a partir de los trabajos de Richard M. Buke, Karl Jung y Abraham Maslow entre otros y cuya línea de acción integra los aspectos espirituales y trascendentes de la experiencia humana. Estos van más allá del ámbito de la mente, estableciendo una mirada integradora de las diferentes perspectivas psicológicas, que permite trascender interpretaciones 
parciales con respecto a la realidad y a la naturaleza de la experiencia humana. Cabe señalar que dentro de esta vertiente de la psicología, son claves los conceptos de conciencia superior, trances sistémicos y estados de interconexión y unificación espiritual, lo cual guarda cierta vinculación con algunos aportes del nuevo paradigma científico y el pensamiento sistémico que hemos expuesto en apartados anteriores.

\section{La educación en el marco del nuevo paradigma}

El enfoque sistémico en el marco de la educación toma en consideración aquellos aspectos de la realidad intangible y global, que usualmente suelen pasar por alto los enfoques reduccionistas. En materia de educación, De la Fuente y Monroy (1995) señalan que tomar la decisión consciente de hacer uso de estos principios nos posibilita, conjuntamente con las otras características sistémicas, cometer menos errores, aprender de estos y aumentar los aciertos.

Comprender como un sistema al entramado de fenómenos sociales y humanos implicados en la educación permite mirar con más claridad tanto sus respectivas partes e interrelaciones, como la dinámica general del todo que los compone, conformándose así una visión holística - gestalt que engloba e integra. Desde esta óptica, De la Fuente y Monroy (1995) sostienen que el percibir la realidad y la educación como sistemas resulta indispensable, ya que solo así se integrarán lo tangible y lo intangible, lo formal e informal, el individuo y su contexto, el cuerpo y la mente, lo somático y lo psíquico, lo psicológico y lo espiritual y; a nivel de contexto, lo natural y lo artificial, lo cultural y lo social, lo eco-social y lo socio-técnico. Los mismos autores afirman que, de esta manera, se podrían establecer las bases para la construcción de un enfoque más humanístico y transpersonal de la educación.

Sin duda, la visión global e integradora, que el nuevo paradigma asume, sugiere rutas, también en materia de política educativa, que comprendan la naturaleza humana desde una perspectiva holística, a partir de "sistemas" interdependientes, que conectan los aspectos biológicos, psicológicos, sociales y espirituales del individuo. Este tipo de iniciativas debería integrar, de manera intencional, la enseñanza explícita de todo tipo de competencias genéricas relacionadas con la educación para la vida, la espiritualidad, el autoconocimiento y la educación emocional. 
Desde un marco de referencia próximo a estas aportaciones, Bisquerra (2012) establece una comparación entre diversos principios que rigen un tipo de educación basada en el viejo paradigma y una educación, de corte trascendental y emocional, cimentada en el nuevo paradigma. Estos se presentan en la tabla 3.

Tabla 3. Diferencias entre antiguo y nuevo paradigma en la educación

\begin{tabular}{|l|l|}
\hline \multicolumn{1}{|c|}{ ANTIGUO PARADIGMA } & \multicolumn{1}{c|}{ NUEVO PARADIGMA } \\
\hline Materialismo & Valores \\
\hline Trabajar para tener & Trabajar para fluir \\
\hline Bienestar objetivo & Bienestar emocional \\
\hline Cognición & También emoción \\
\hline Letra con sangre entra & Enseñar deleitando \\
\hline Emociones negativas & Emociones positivas \\
\hline
\end{tabular}

Nota: Diferencias entre antiguo y nuevo paradigma (Bisquerra, 2012).

Estos principios están fuertemente comprometidos con el desarrollo de una percepción de la educación, como imbuida dentro de una red compleja de sistemas que pretenden, tal y como lo establecía su raíz latina "educere", sacar del individuo su verdadera naturaleza humana, la verdad, la belleza, la plenitud, el autoconocimiento y en general todos aquellos aspectos que lo trasciendan y para lo cual también es preciso comprender la sociedad y la educación desde una perspectiva estética, socio histórica, estructural y hermenéutica, que genere políticas educativas compatibles con la complejidad que el sistema educativo demanda en estos términos.

Desde esta perspectiva, las innovaciones educativas que se desarrollen tendrán que ser planteas desde una visión de ecología profunda, que comprenda y dinamice el funcionamiento de las partes, sus respectivas interrelaciones y las características generales de las condiciones y contexto global en el cual se pondrán en marcha.

\section{Innovación educativa: Conceptos generales}

$\mathrm{Al}$ analizar el término innovación, vemos que su origen proviene del latín "innovatio", cuyo significado del prefijo "in", de ascendencia latina, equivale a: en, adentro, dentro de, al interior. En este caso, el 
prefijo "in" aporta una connotación natural de interioridad, entendido como introducción de algo novedoso, a partir de condiciones ya presentes en el contexto donde se implementará dicha innovación. Por su parte, Rimari (2003) sostiene que una innovación sería definida como el ingreso de algo nuevo, dentro de una realidad preexistente. $\mathrm{Al}$ respecto, algunos estudios, tales como el de Monereo (2007), recomiendan no introducir innovaciones educativas muy alejadas de la realidad del centro educativo o proyecto académico, sino que estas mismas surjan a partir de iniciativas ya planteadas por la institución y que reconozcan la complejidad de las dinámicas particulares y generales que giran en torno a las problemáticas que pretenden atender.

Así pues, son muchos los autores y autoras que han aportado distintas concepciones del término innovación educativa. Al respecto, Imbernón (1996) plantea:

La innovación educativa es la actitud y el proceso de indagación de nuevas ideas propuestas y aportaciones, efectuadas de manera colectiva, para la solución de situaciones problemáticas de la práctica, lo que comportará un cambio en los contextos y en la práctica institucional de la educación. (p. 64)

De lo anterior, se asume que la implementación de toda innovación educativa obedece a la calidad de las percepciones, profundas o superficiales, sobre las cuales se interpreta un contexto educativo en el cual se desee introducir una innovación. También se requiere que esta esté ligada a proyectos estratégicamente articulados desde adentro de la institución, con el propósito de garantizar su continuidad en el largo plazo.

\section{Objetivos generales de la innovación educativa}

Desde este marco conceptual, pensar en innovación educativa supone estrictamente el desarrollo del conocimiento condicional o estratégico, que permita planificar objetivos concretos ajustados a la complejidad que el contexto de innovación demanda. Así pues, Rimari (2003) sugiere algunos objetivos generales que merece la pena tener en consideración de cara al diseño de innovaciones en el ámbito educativo. Estos se comentan a continuación: 
a. Desarrollar actitudes positivas en toda la comunidad educativa en función de un comportamiento permanente, abierto a la necesidad del cambio y sus implicaciones, a la adecuación del currículo y a las necesidades e intereses de los alumnos y alumnas.

b. Establecer espacios y mecanismos en las instituciones educativas para identificar, valorar, sistematizar, normalizar, aplicar y difundir las experiencias novedosas que contribuyan a la solución de problemas educativos que estén afectando la calidad de los aprendizajes del estudiantado.

c. Propiciar el desarrollo de propuestas educativas válidas que respondan a la realidad de nuestro país y que rescaten la creatividad, la riqueza humana y los recursos naturales y culturales que provee nuestro medio.

d. Promover transformaciones curriculares flexibles, creativas y participativas, acordes con las necesidades de los sujetos y de su comunidad, procurando una educación de calidad y aprendizajes significativos.

e. Implementar la aplicación de teorías, procesos, métodos y técnicas administrativas y docentes reconocidamente válidas, congruentes con las necesidades de la institución y de la comunidad, en su propósito de buscar una mejor calidad de la educación.

f. Estimular la investigación como un elemento cotidiano determinante de la formación profesional continua de docentes a partir de su propia práctica educativa.

g. Recuperar y sistematizar experiencias del personal docente, directivo, asesor y supervisor.

h. Compartir y transferir a otras escuelas y docentes las experiencias educativas innovadoras para ampliar y generalizar la experiencia.

i. Crear condiciones permanentes para que las experiencias innovadoras se conviertan en una práctica institucionalizada, es decir, en cultura organizacional.

Como puede observarse, los anteriores objetivos sugieren claramente la necesidad de comprender, desde una perspectiva de ecología profunda, todas las posibles variables que interactúan en un contexto educativo en que se planifique introducir una innovación. También se evidencia un fuerte compromiso con el hecho de que las iniciativas de innovación puestas en marcha se conviertan progresivamente 
en prácticas institucionalizadas, que formen parte de la cultura organizacional del centro educativo, para lo cual, como se ha señalado, es imprescindible el desarrollo de una percepción global de la dinámica organizacional en que será introducida una determinada innovación.

\section{Principios generales de la innovación educativa}

Los principios que sustentan la innovación educativa pueden desarrollarse con mayor facilidad dentro de un tipo de educación flexible y holística, que comprenda al ser humano desde su dimensión biológica, psicológica, social y espiritual. Las aportaciones señaladas por Rimari (2003) sostienen que los fundamentos que orientan el proceso de innovación educativa solo se pueden desarrollar dentro de un marco de educación liberadora de las potencialidades del ser humano y de su entorno. El mismo autor establece los siguientes principios:

a. La formación del estudiantado constituye la esencia de las innovaciones educativas para la transformación cultural en procura de mejorar el nivel de vida individual y social.

b. La autonomía es básica para que se generan los procesos de innovación educativa.

c. La investigación interdisciplinaria se requiere para la reconstrucción del conocimiento, como eje proceso de innovación.

d. La práctica misma legitima la innovación educativa.

Es evidente que la transformación cultural, la autonomía de individuo y la reconstrucción del conocimiento, entre otros, representan los principios de innovación base sugeridos por el autor, colocan al ser humano como centro y principal beneficiario de toda iniciativa de innovación llevada a la práctica. Por ello, para efectos de los objetivos de este artículo, podemos definir la innovación educativa como aquellas iniciativas, acciones o políticas educativas, que tras un análisis sistémico de las condiciones generales del contexto, pretendan diagnosticar, analizar, evaluar o atender problemas prototípicos ó emergentes relacionados con la educación. 


\section{Percepción sistémica de la innovación educativa}

La percepción sistémica de la educación, entendida como la comprensión respecto al funcionamiento de sus componentes, sus interrelaciones y la dinámica general asumida por el sistema que la nutre, representa la clave para que determinadas innovaciones educativas sean planificadas desde una perspectiva intencional y deliberada y con esto cumplan el objetivo para el cual fueron creadas. En esta línea, De la Fuente y Monroy (1995) sugieren una serie de características que la innovación educativa debería enfatizar en la era sistémica. Estas se exponen a continuación:

a. $\quad$ El aprendizaje debe enfatizar no solo aspectos vinculados con la enseñanza, sino también aquellos relacionados con la motivación de aprender y el desarrollo de habilidades orientadas a aprender a aprender a través de la acción.

b. Se deben promover la enseñanza explicita de conductas de adaptación, con el propósito de integrar la secuencia: percepción, pensamiento y acción en la transformación individual y circunstancial. En este sentido es importante pensar en términos de una adaptación activa en vez de la adaptación pasiva, en la que el sujeto solo suele cambiar con el propósito de adaptarse a los cambios que el contexto le impone. Desde esta óptica, la adaptación activa sugiere al individuo el desarrollo de mayores competencias de flexibilidad.

c. Es importa también la inclusión de plataformas de educación continua que permitan al sujeto aprender de manera permanente y a lo largo de la vida y transferir esos aprendizajes a diferentes situaciones y contextos.

Quizá uno de las características generales de la innovación educativa sistémica, y que es compatible con lo citado anteriormente, corresponde al hecho de que toda innovación deberá garantizar el aprendizaje de herramientas que permitan al estudiantado gestionar su propio aprendizaje y conocer los diferentes contextos reales de aplicación en que tienen lugar. De esta manera, el aprendiz podrá atribuir con claridad un sentido y significado inmediato a lo que aprende. 


\section{Conclusiones}

Así como el nuevo paradigma científico lo propone, toda iniciativa de innovación educativa deberá priorizar acciones que atiendan problemáticas globales y las distintas dinámicas subyacentes que los posicionamientos reduccionistas tienden a desestimar. Dichas innovaciones, lejos de centrarse en aspectos superficiales del problema, desde visiones antropocéntricas y competitivas, deberán comprender el proceso de innovación desde una cosmología más amplia, en donde la cooperación y la interdependencia positiva se constituyan en la base articuladora de toda experiencia que pretenda establecer acciones de innovación en cualquier área de la educación.

Esta visión sistémica de las innovaciones en el campo de la educación y de la profundidad de los múltiples desafíos y escenarios que pretenden atender contribuye, radicalmente, en el desarrollo de una percepción más amplia y holística de la complejidad subyacente de los diversos problemas prototípicos y emergentes que giran en torno a la educación.

Si la educación representa una de las principales plataformas para el desarrollo humano, desde este marco conceptual, toda iniciativa de innovación educativa deberá contemplar una visión de desarrollo humano integral, ya que entiende al ser humano y el fenómeno de la vida desde una perspectiva más amplia y global. Lo anterior, se visualiza en la capacidad que estos principios poseen de integrar, complementar y relacionar los aspectos base que conforman la identidad del ser humano, en ámbitos de carácter biológico, psicológico, social y espiritual. Las conclusiones obtenidas de las reflexiones realizadas en torno a esta temática han aportado claves fundamentales para comprender el sentido de la vida, lo cual ha resultado, en muchos casos, compatible con algunas enseñanzas filosóficas y tradiciones espirituales antiguas orientadas hacia una mejor comprensión de lo trascendente de la experiencia humana.

En síntesis, optar por una visión sistémica de la innovación educativa supone, en definitiva, la resolución de un problema de percepción, concretamente arraigado en el funcionamiento de las partes de los fenómenos educativos. Lo anterior, implica un fuerte compromiso de asumir una posición de conciencia respecto al "mapa" más allá del "territorio", y de las posibilidades que podría brindar esta visión en el desarrollo de una nueva ecología del aprendizaje. Una nueva ecología comprometida con un modelo distinto del modelo de escolarización tradicional y que 
percibe los fenómenos implicados en la educación desde una posición más global, reconociendo la relevancia que en la actualidad han tomado los diversos escenarios de aprendizaje fuera del contexto escolar. Estos principios son coherentes con las aportaciones de Coll (2013), el cual señala que esta nueva ecología del aprendizaje supondría generar un tipo de educación constituida a través de relaciones estrechas entre la escuela, la familia y otros contextos de educación no formales, lo cual brinda oportunidades de aprendizaje en las que subyacen redes interconectadas de escenarios cada vez más diversos que permitirán, a su vez, el aprender a lo largo y a lo ancho de la vida; principios que aportan ideas valiosas y compatibles con las reflexiones en torno a la percepción sistémica de la innovación expuesta en este articulo.

\section{Referencias}

Bisquerra, R. (2005). La educación emocional en la formación del profesorado. Revista Interuniversitaria de Formación del Profesorado, 19(3), 95-114.

Bisquerra, R. (2012). La educación emocional: Factor clave del nuevo paradigma. IX Congreso Mundial de Educación ACADE. Recuperado de https://www.youtube.com/watch?v=sGaAByfrZ8A

Coll, C. (2013). El currículo escolar en el marco de la nueva ecología del aprendizaje. Aula, 219, 31-36.

Bohr, N. (1934). Atomic Physics and the Description of Nature. Cambridge University Press, Londres.

Capra, F. (1988). La trama de la vida. Barcelona: Editorial Anagrama.

Capra, F. y Steindl-Rast, D.. (1993). Pertenecer al universo. Buenos Aires: Editorial Planeta.

Castanedo, C. (1997). Terapia gestalt, enfoque centrado en el aquí $y$ ahora $\left(3^{\circ}\right.$ Ed.). Barcelona: Herder.

De la Fuente, E. y Monroy, S. (1995). Un concepto sistémico de innovación educativa Cuadernos de Reencuentro, 14, 6-10.

Imbernon, F. (1996). En busca del discurso educativo. La escuela, la innovación educativa, el curriculum, el maestro y su formación. Buenos Aires, Argentina, Magisterio del Río de la Plata.

Lipton, B. (2009). The Biology of Belief. San Francisco State University: SFSU. Recuperado de http://www.sfsu.edu/ holistic/Welcome.html 
Maturana, H. y Varela, F. (1980). Autopoiesis: The organization of the living. Santiago de Chile: Editorial Universitaria.

Merlo, V. (2007). La reencarnación, claves para entender el sentido de la vida: Concepciones antiguas y modernas. España: Editorial Sirio.

Monereo, C. (2007). La evaluación auténtica de competencias: Posibles estrategias. Recuperado de http://www.sinte.es/ carlesmonereo/?page id=101

Naess, A. (1973). The shallow and the deep, long range ecology movements. Recuperado de http://www.alamut.com/subj/ideologies/ pessimism/Naess_deepEcology.html

Rimari, W. (2003). Guía para la formulación de proyectos de innovación educativa. Lima, Perú: Asociación Cultural San Jerónimo.

Rindaldi, S. (2003). El Kybalion y la Ciencia. Buenos Aires: Editorial Kier. Roberts, J. (1998). Habla Set 1: La eterna validez del alma. Barcelona: Editorial Luciérnaga.

Sahtouris, E. (1994). Gaia, la tierra viviente. Buenos Aires: Editorial Planeta. 